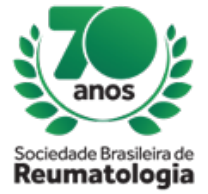

CENTRO DE EVENTOS DO CEARÁ O4 A 07 DE SETEMBRO

\title{
A FOREHEAD ULCER IN A PATIENT WITH SYSTEMIC LUPUS ERYTHEMATOSUS
}

Camila de David Cruz (Serviço de Reumatologia do Hospital Nossa Senhora da Conceição - Grupo Hospitalar Conceição, Porto Alegre, RS, Brasil), Sheron Zamboni (Serviço de Reumatologia do Hospital Nossa Senhora da Conceição - Grupo Hospitalar Conceição, Porto Alegre, RS, Brasil), Clarice Gabardo Ritter (Serviço de Dermatologia do Hospital Nossa Senhora da Conceição - Grupo Hospitalar Conceição , Porto Alegre, RS, Brasil), Vanessa Barrili Busato (Serviço de Reumatologia do Hospital Nossa Senhora da Conceição - Grupo Hospitalar Conceição, Porto Alegre, RS, Brasil), Romulo Gomes Silveira (Serviço de Reumatologia do Hospital Nossa Senhora da Conceição - Grupo Hospitalar Conceição , Porto Alegre, RS, Brasil), Valentina Oliveira Provenzi (Serviço de Patologia do Hospital Nossa Senhora da Conceição Grupo Hospitalar Conceição, Porto Alegre, RS, Brasil), Markus Bredemeier (Serviço de Reumatologia do Hospital Nossa Senhora da Conceição - Grupo Hospitalar Conceição, Porto Alegre, RS, Brasil)

\section{BACKGROUND}

Epstein Baar virus-positive mucocutaneous ulcer (EBVMCU) is part of the spectrum of B-cell lymphoproliferative diseases, and can affect the oropharyngeal mucosa, skin and digestive tract. It was first described in 2010 by Dojcinov et al. in 26 patients, 9 of them under treatment with metotrexate, azathioprine or cyclosporine. The disease usually presents as a well delimited, unique ulcer of slow evolution. Risk factors are advanced age and iatrogenic immunosuppression. It was recognized as a new clinicopathological entity in the review of the World Health Organization (WHO) in 2017. The major differential diagnoses are lymphomatoid granulomatosis and EBV-positive diffuse large B-cell lymphoma. The course of EBVMCU is generally indolent and self-limiting. Treatment is conservative and involves reduction of dose or interruption of immunosupressants.

\section{CASE REPORT}

A 47 years-old afro-brazilian woman with diagnosis of systemic lupus erythematosus (SLE) presented to medical consultation in March 2018. She reported the emergence of an otherwise asymptomatic nodular lesions on the right side of the forehead $(1.2 \mathrm{~cm}$, Figure 1$)$ and in the left thigh $(3.0 \mathrm{~cm})$ starting 2 months ago. The patient was then forwarded for incisional skin biopsy. In the past (2001-2002), she had been treated with intravenous cyclophosphamide for nephritis and was currently receiving treatment with methotrexate $25 \mathrm{mg} /$ week, azathioprine $200 \mathrm{mg} /$ day, hydroxychloroquine $400 \mathrm{mg} /$ day and prednisone 5 $\mathrm{mg} /$ day due to persistent lupus activity. By the end of May 2018, the forehead lesion had evolved with rapid growth $(3.5 \mathrm{~cm})$ and ulceration. Biopsy of this lesion showed atypical cell proliferation of an indeterminate nature. A few weeks latter, the immunohistochemical results of the biopsy were available showing a lymphoproliferation of large B-cells, EBV-positive, with a marked T-cell background (atypical cells were positive for CD3, CD20, CD30, MUM1, and EBV). An extensive additional investigation including computed tomography of head, neck, chest, and abdomen did not demonstrate lymphadenopathies, mass lesions in internal organs, or hematologic abnormalities, confirming the diagnosis of EBV-positive mucocutaneous ulcer (EBVMCU). Treatment with methotrexate and azathioprine was interrupted, and the lesions progressively improved over the next 2 months.

\section{CONCLUSION}

The present case represents the rare ocurrence of a recently described clinical entity in a Brazilian patient with SLE. 